\title{
Early antecedents of suicide: the role of prenatal and childhood risk factors
}

\author{
Jussi Jokinen
}

Karolinska Institutet, Department of Clinical Neuroscience, Karolinska Institutet, Building R5 Karolinska University Hospital, Stockholm SE-17176, Sweden; jussi.jokinen@ki.se

\section{WHAT IS ALREADY KNOWN ON THIS TOPIC?}

Childhood trauma plays a significant role in the development of vulnerability to suicidal behaviour. ${ }^{\text {In }}$ In prospective and record linkage studies, adverse prenatal and perinatal circumstances and behavioural problems in childhood have been associated with suicidal behaviour later in life. ${ }^{2}{ }^{3}$ Clinical research on suicide attempters indicates that assessment of both childhood trauma and violent behaviour may detect patients at suicide risk. ${ }^{1}$

\section{WHAT DOES THIS PAPER ADD?}

- Results from a 50-year follow-up of a large birth cohort with information on both prenatal circumstances and on developmental and adversity factors recorded at the age of 7 years, in relation to suicide death in adulthood, report associations between several early risk factors and later suicide, suggesting that trajectories leading to suicide death start at early life.

- Both prenatal factors, such as low birth weight, younger maternal age, higher birth order, and emotional adversity in the form of parental death, bullying by peers and externalising problems were associated with higher suicide risk.

- The associations between prenatal risk factors and suicide were not explained by emotional adversities or behavioural problems experienced up to the age of 7 .

\section{LIMITATIONS}

- Owing to the small number of suicides, the study lacks powerespecially concerning analyses of sex differences.

- The researchers did not have information of abuse in childhood. Abuse in childhood has been repeatedly related to suicidal behaviour in adulthood. ${ }^{1}$
- Information of eventual psychiatric illness in adulthood was not included in the mediation analyses. It is important to know if the vulnerability to later suicidal behaviour is associated with prenatal and childhood risk factors independently of psychiatric illness.

\section{WHAT NEXT IN RESEARCH?}

- To focus on underlying neurobiological mechanisms, like stress regulation and oxytocin system related to prenatal and early adversity.

- Randomised controlled studies with long-term follow-up should assess the effects of early interventions to prevent later suicidal behaviour.

\section{CAN THESE RESULTS CHANGE YOUR PRACTICE AND WHY?}

Yes, since this study raises awareness of the association between early adversity and externalising problems, and later risk of suicide. Early detection and interventions to prevent externalising problems and aggressive behaviour are important clinical implications of this study. In adult psychiatry, exposure to both childhood trauma and violent behaviour should be part of the clinical suicide risk assessment to detect patients at high long-term suicide risk. ${ }^{1}$

Competing interests None.

doi:10.1136/eb-2014-101930

\section{REFERENCES}

1. Jokinen J, Forslund K, Ahnemark E, et al. Karolinska Interpersonal Violence Scale predicts suicide in suicide attempters. J Clin Psychiatry 2010;71:1025-32.

2. Mittendorfer-Rutz $\mathbf{E}$, Rasmussen F, Wasserman D. Restricted fetal growth and adverse maternal psychosocial and socioeconomic conditions as risk factors for suicidal behaviour of offspring: a cohort study. Lancet 2004;364:1135-40.

3. Klomek AB, Sourander A, Niemelä S, et al. Childhood bullying behaviors as a risk for suicide attempts and completed suicides: a population-based birth cohort study. J Am Acad Child Adolesc Psychiatry 2009;48:254-61.

ABSTRACT FROM: Geoffroy MC, Gunnell D, Power C. Prenatal and childhood antecedents of suicide: 50-year follow-up of the 1958 British Birth Cohort Study. Psychol Med 2013;29:1-12.

Patients/participants The 1958 birth cohort, including 98\% of people born in England, Scotland and Wales during 1 week in March 1958 ( $n=16470)$. People who subsequently emigrated were excluded. Data were obtained from a birth survey, a survey when they were 7 years old and death certificates. Complete data were available for 12399 people.

Setting UK.

Exposure Low birth weight, gestational age, maternal smoking during pregnancy, mother's age at birth and the child's birth order; all were reported by participants' mothers when participants were 7 years old. The survey at age 7 included school tests for maths and reading, behaviour problems assessed by teachers and mothers, bladder control, and socioeconomic adversity. Emotional adversity was defined as parental death, divorce/separation, domestic tension, contact with social services, time spent in institutional care, or signs of neglect or bullying. Follow-up period 31 May 2009, or date of death if prior to 2009.

\section{OUTCOMES}

Incidence of suicides Fifty-one of 1475 deaths (44 males and 7 females). The median age at suicide was 40 for men and 39 for women.

Prenatal risk factors associated with suicide Birth weight less than $2.5 \mathrm{~kg}$ increased risk ( $\mathrm{HR}=2.47,95 \%$ CI 1.05 to 5.81$)$. Maternal age over
29 years at child's birth decreased risk compared to maternal age of 20 29 years ( $\mathrm{HR}=0.44,95 \%$ CI 0.21 to 0.92 ).

Developmental risk factors associated with suicide Being wet during the day after age 3 years increased risk ( $\mathrm{HR}=2.72,95 \%$ CI 1.08 to 6.88). Teacher-assessed externalising behaviours, described as 'intermediate' ( $\mathrm{HR}=2.23$, 95\% CI 1.10 to 4.49 ) and 'problem' (HR=2.67, 95\% CI 1.15 to 6.18 ) increased risk compared to no problems. Maternal-assessed problem externalising behaviour also increased risk $(\mathrm{HR}=2.89,95 \% \mathrm{CI} 1.24$ to 6.71). Cognitive ability or internalising behavioural problems were not associated with risk.

Indicators of adversity associated with suicide Parental death ( $\mathrm{HR}=4.52,95 \%$ CI 1.40 to 14.59$)$, institutional care ( $\mathrm{HR}=4.22,95 \% \mathrm{CI} 1.51$ to 11.76 ), contact with social services ( $\mathrm{HR}=1.80,95 \% \mathrm{CI} 1.17$ to 2.76 ), and frequent peer bullying ( $\mathrm{HR}=2.84,95 \% \mathrm{CI} 1.16$ to 6.96 ) all increased risk

Overall number of emotional adversities Three or more adversities increased suicide risk compared to none ( $\mathrm{HR}=5.27,95 \% \mathrm{CI} 1.85$ to 14.99 ).

All prenatal and postnatal factors assessed on multivariate analysis Significant associations remained only for birth weight less than $2.5 \mathrm{~kg}$ $(\mathrm{HR}=2.48)$, maternal age over 29 years $(\mathrm{HR}=0.41)$, and three or more emotional adversities $(\mathrm{HR}=3.12)$. 